\title{
tRNA-Derived Fragments in Seminal Plasma Exosomes Are Non-invasive Biomarkers for Diagnosis of Non-obstructive Azoospermia With Spermatogenic Failure
}

Zuobin Zhu ( $\sim$ zhuzuobin@xzhmu.edu.cn )

Xuzhou Medical University https://orcid.org/0000-0003-1022-5943

Xiaoxiao Han

School of Life Science, Tongji University

Ying Li

Xuzhou Medical University

Yao Zhu

Xuzhou Medical University

Zhenbei Li

Xuzhou Central Hospital

Qiang Zhang

Xuzhou Medical University

Liang Wang

Xuzhou Medical University

Hongjin Chen

Xuzhou Medical University

Lin Hao

Xuzhou Central Hospital

Fangfang $\mathrm{Hu}$

Xuzhou Central Hospital

Yijuan Cao

Xuzhou Central Hospital

Conghui Han

Xuzhou Medical University

\section{Research}

Keywords: tsRNA, non-obstructive azoospermia, obstructive azoospermia, biomarker

Posted Date: October 14th, 2020 
DOI: https://doi.org/10.21203/rs.3.rs-89955/v1

License: (c) (i) This work is licensed under a Creative Commons Attribution 4.0 International License. Read Full License 


\section{Abstract}

\section{Background}

There is lack of accurate and non-invasive preoperative evaluation for improving microdissection testicular sperm success rate. tRNA-derived small RNA (tsRNAs) perform a variety of physiological functions and are related to many physiological and pathological processes. This study sought to identify the potential of exosomal tsRNA as a novel non-invasive diagnostic biomarker for nonobstructive azoospermia (NOA) with spermatogenic failure.

\section{Methods}

Seminal plasma exosome tsRNA levels were used in a two-stage (screened by tsRNA sequencing on Illumina NextSeq instrument and validated by qRT-PCR) case-control designed study. The expression levels of the selected tsRNAs were further examined in the testicular tissues of NOA patients and obstructive azoospermia (OA) patients, and their underlying role in the pathogenesis of non-obstructive azoospermia was performed by bioinformatic analysis.

\section{Results}

In this study, two tsRNAs (tRF-Val-AAC-010: AUC $=0.96$, specificity $=80 \%$, sensitivity $=95 \%$; tRF-Pro-AGG003: $A U C=0.96$, specificity $=87 \%$, sensitivity $=95 \%$ ) were found to have a good predictive accuracy which also showed differential expression in testicular tissue between NOA patients and OA patients. We also found that the combinations of tRF-Val-AAC-010 and tRF-pro-AGG-003 have a better discriminating ability between NOA patients and OA patients than single biomarkers. Finally, the bioinformatic analysis showed that the two selected tsRNAs were involved in spermatogenesis.

\section{Conclusion}

This study first evaluated tsRNAs as potential biomarkers for NOA diagnosis and identified the exosomal tRF-Val-AAC-010 and tRF-pro-AGG-003 in seminal plasma valuable as biomarkers for NOA diagnosis.

\section{Background}

As a reproductive system disease, infertility affects about $15 \%$ of the world's population[1] among which the male factor accounts for about $50 \%$ [2]. The incidence of azoospermia accounts for $10 \% \sim 20 \%$ of male infertility. According to the presence or absence of sperm in the testes, it can be classified into two groups: one that is obstructive azoospermia (OA) with normal spermatogenesis, and the sperm could be obtained from microdissection testicular sperm extractions (TESE); the other that is non-obstructive azoospermia (NOA) which accounts for $60 \%$ of azoospermia, and the sperm could be obtained from microdissection testicular sperm extractions (TESE). An accurate diagnosis of non-obstructive azoospermia could provide valuable guidance to clinicians. Although conventional biochemical markers in semen, such as citric acid, acid phosphatase, fructose, and alpha-glucosidase can be used to diagnose 
OA patients; and testicular volume, serum follicle-stimulating hormone (FSH) levels can be used to diagnose NOA patients, was however, a proportion of NOA and OA patients that cannot be diagnosed correctly by using these markers still exists[3].

The pathological diagnosis of testicular biopsy is the "gold standard" for determining spermatogenesis status in the testis[4]. However, invasive procedures not only cause pain to patients but also may cause progressive and irreversible destruction and structural distortion of spermatogenic tubules $[4,5]$. Accurate and non-invasive preoperative evaluation is of great significance for improving TESE success rate, reducing unnecessary surgical trauma, and reducing patients' psychological burden. In this study, we aim to identify biomarkers which can distinguish the etiology of azoospermia or can accurately evaluate patients who will have a positive or negative testicular sperm extraction. Exosomes are extracellular vesicles secreted by most cells with a diameter of $30 \sim 150 \mathrm{~nm}$ and a lipid bilayer membrane structure. The testis of patients with non-obstructive azoospermia is not capable of producing sperm, and its tissue structure is significantly different from that of patients with obstructive azoospermia, which is capable of producing sperm[4]. Therefore, exosomes in seminal plasma produced by NOA patients and OA patients are also different. Thus, seminal plasma exosomes are emerging as a source of potential biomarkers for the pathological and physiological conditions of the testis.

A recent study showed that exosomal miRNAs in seminal plasma are excellent markers for predicting the presence of sperm in the testis[3]. In recent years, a novel regulatory small non-coding RNA, called tRNAderived small RNA (tsRNA), has been found to be widely enriched in human body fluids[6]. tsRNAs are fragments which are produced by precise regulated processing of tRNA and its precursors and can be divided into two categories: tRNA related fragments (tRF) and tRNA halves (tiRNA), with nucleotide compositions, characteristic sizes, functions, and biogenesis[7]. tsRNAs can also be classified into a variety of different types depending on where they map on the mature or precursor tRNA transcript[8]. According to their mapped positions, these tsRNAs are divided into five types: tiRNA, tRF-1, tRF-2, tRF-3 and tRF-5[8, 9].

It has been reported that tsRNAs perform a variety of physiological functions and are related to many physiological and pathological processes[10]. The composition and number of tsRNAs are highly dependent on specific cell types and pathological conditions, making them an excellent class of biomarkers. tsRNAs have become a cancer survival indicator and a potential diagnostic marker[11]. Recent studies found that tsRNAs are expressed in testicular and sperm cells[12,13] and are conserved in gametes among mammals[14]. tsRNA can also tightly bound to Piwi protein which plays an important role in maintaining the normal function of germline stem cells[15]. Since tsRNAs play an important role in sperm maturation and fertilization in mammals $[14,16]$, and they are higher enriched in seminal plasma and exosomes than miRNA, suggesting that tsRNA can be used as a promising biomarker for NOA diagnosis. The role of tsRNAs in the origin of azoospermia remains unclear. This study aims to evaluate the predictive power of tsRNAs in testicular sperm extraction for patients with azoospermia. 
Since NOA is affected by multiple biologically redundant factors, the combination of multiple biomarkers could also contribute to discriminate NOA patients from OA patients. For many common diseases, polygenic risk scores of multiple common variations have better disease risk prediction than single rare or common mutations 9,10 . The previous study also showed that the collective effects of common SNPs in which single variation has small effect sizes in diseases could improve risk prediction for many diseases $[17,18]$. Generalized linear model (GLM) is a good predictor since it is known to lead to highly accurate predictions[19]. In this study, the GLM was employed to determine the optimal combinations of tsRNAs for predicting the presence of sperm in testicular tissue.

\section{Materials And Methods}

\section{Subjects of study}

23 NOA patients, 18 OA patients, 5 idiopathic oligoasthenospermia (IO) patients and 13 healthy people participating in the study were from the Xuzhou Central Hospital. This study was approved by the Research Ethics Commission of Xuzhou Central Hospital and written informed consent was obtained from all participants. In this study, the 23 NOA patients and 18 OA patients have a total absence of sperm in the ejaculate, and the OA patients who have normal spermatogenesis can obtain a sufficient number of sperm for fertilization by TESE, but the NOA patients who have no spermatogenic reserve of the testis have a negative outcome by the TESE. The 10 patients $\left(<10 \times 10^{6} \mathrm{sperm} / \mathrm{ml}\right)$ and healthy people $(>50 \times$ $10^{6} \mathrm{sperm} / \mathrm{ml}$ ) were also included in the study. In this study, all the samples used in the screening and validating stages did not show genetic causes (Y-chromosome microdeletion and chromosomal aberration) and clinical factors (anatomic malformation, varicocele and hemospermia). The baseline clinical disease characteristics are shown in Supplementary Table 2.

\section{Exosome extraction}

Umibio® exosome isolation kits (Umibio, Cat. No: UR52130, China) were used for exosome extraction. In brief, each sample was centrifuged for 10 minutes at $3,000 \mathrm{~g} 4^{\circ} \mathrm{C}$, and $10,000 \mathrm{~g} 4^{\circ} \mathrm{C}$ for 20 minutes to remove cells and debris. Then the reagent was added to the volume of the initial sample according to the manufacturer's instructions. Then the corresponding amounts of reagents were added to sample volume. The mixture was vortexed at $4^{\circ} \mathrm{C}$ for two hours and then centrifuged at $10,000 \times \mathrm{xg} 4{ }^{\circ} \mathrm{C}$ for 60 minutes to precipitate exosome pellets. Pellets were resuspended with $1 \times \mathrm{PBS}$ and purified with Exosome Purification Filter at $3,000 \times \mathrm{xg} 4^{\circ} \mathrm{C}$ for 10 minutes. The Exosome solution was stored at $-80^{\circ} \mathrm{C}$ immediately after isolation until the following analysis.

\section{Library preparation and sequencing}

The library preparation in this study was based on previous studies[20]. The DNA fragments from wellmixed libraries were denatured with $0.1 \mathrm{M} \mathrm{NaOH}$ to produce single-stranded DNA molecules, and they were then added to the reagent cartridge at $1.8 \mathrm{pM}$ concentration. The tsRNA sequencing was carried out 
on a NextSeq system using NextSeq 550 V2 kit (\#FC-404-2005, Illumina) in accordance with the manufacturer's instructions and the sequencing runs for 50 cycles.

\section{Statistical analysis}

In this study, sequencing quality was examined by FastQC and trimmed 5', 3'-adaptor reads by cutadapt were aligned allowing for 1 mismatch only to the mature tRNA sequences[21], then reads that do not map were aligned allowing for 1 mismatch only to precursor tRNA sequences with bowtie software[22]. The remaining reads were aligned allowing for 1 mismatch only to miRNA reference sequences with miRDeep2. The expression profiling of tsRNAs can be calculated based on counts of reads[23] mapped. The differentially expressed tsRNAs were screened based on the counts per million of total aligned reads with R package edgeR[24]. Principal Component Analysis (PCA), Pie plotsand Volcanoplots were performed on R-Studio version 1.2.5033 for statistical computing and graphics of the expressed tsRNAs.

In this study, the candidate tsRNAs were screened by the deep sequencing technology in small number of samples, and at the same time, they should meet the follow conditions: (1) The tsRNAs should show statistically significant differential expressions by both parametric test (Student's t-test) and nonparametric test (negative binomial distribution test) ( $p$-value $<0.05$, fold change $>3$ ); (2) The tsRNAs of each sample in one group should be expressed higher or lower than each individual in the other group. The selected differentially expressed tsRNAs were verified by qRT-PCR. The "Area Under ROC Curve (AUC)", the value of sensitivity and specificity were performed using the GraphPad Prism7. The means and standard error are used to represent the continuous variables. The negative binomial distribution test and Student's t-test were used, where applicable. Generalized linear models (GLM) and Pearson correlation test were performed on R-Studio version 1.2.5033. GLM covariates are selected using binomial regression and the best fit subset using the Bayesian information criterion (BIC).

\section{Results}

\section{Profiling of exosomal tsRNAs in seminal plasma}

To identify tsRNA as a promising biomarker for NOA, we first isolated exosomes from seminal plasma and prepared tsRNA library for high throughput sequencing. Since tsRNAs are decorated by RNA modifications that interfere with tsRNA-seq library construction[25], total RNA extracted from exosomes are pretreated to get rid of some RNA modifications which interfere with tsRNA-seq library construction. Then the selected tsRNAs as biomarkers were further verified in a subsequent set of blood serum and seminal plasma (Fig. 1A) by RT-qPCR and bioinformatic analysis. The prepared seminal plasma exosomes were confirmed by transmission electron microscopy measurement (Fig 1B) and nanoparticle tracking analysis (Fig 1C).

tRF-5 is the most abundant tRNA-derived small RNA in exosomes (56\%), whereas tRF-2, tRF-1, and tiRNA3 only account for $2.9 \%$ (Fig. 1D). We also analyzed the origin of these tsRNAs and found that the origin for tsRNAs in seminal plasma was distributed in 22 tRNAs (Fig 1E) which was different from tsRNAs 
distribution in plasma such that almost all tRNA-5s derive from four tRNAs (Gly-, Lys-, Glu- and Val-tRNA) [11]. The distributions of 9 tsRNAs were further analyzed, and it was found that the distribution of each type of tsRNA exhibited significant bias. Peculiarly, a large majority of tRF-5c were enriched in five tRNAs (Glu-, Gly-, His-, Lys- and Pro-tRNA); while tRF-1s were mainly enriched in Ser-TGA tRNA (Fig. 1F). The length distributions of tsRNAs were then analyzed and it was found that tRF-1 were 15-21 nt, tRF-2 were $14 \mathrm{nt}$, tRF-3a were 17-18 nt, tRF-3b were 19-22 nt, tRF-5a were 14-16 nt, tRF-5b were 22-24 nt, tRF-5c were 28-32 nt, tiRNA-3 were $40 \mathrm{nt}$ and tiRNA-5 were 33-35 nt (Fig 1G). These results suggested that tsRNAs were not the product of random explanations from tRNA, but may regulate biological function through an unknown mechanism.

\section{Identification of differentially expressed tsRNAs between NOA and OA patients}

To comprehensively profile exosomal tsRNAs among NOA patients, OA patients, and healthy people, we first used Principal Component Analysis (PCA) method which was an unsupervised analysis to reduce the dimensionality of large data sets, and a distinguishable tsRNAs expression profiling among three groups was found (Fig 2A), indicating that the function of exosomal tsRNAs maybe associated with spermatogenesis and it is also effective to select markers from the tsRNAs profile that can distinguish between NOA patients and OA patients. We then screened the significantly differentially expressed tsRNAs between NOA patients and OA patients and found that 38 tsRNAs up-regulated and 46 tsRNAs down-regulated (difference of more than 3-fold change, $P<0.05$ ) (Fig 2B). Then we found that 100 tsRNAs were differentially expressed between NOA patients and healthy people (16 up-regulated, 84 down-regulated) (Fig 2C).

\section{tRF-Val-AAC-010 and tRF-Pro-AGG-003 are the biomarkers of NOA and associated with spermatogenesis status}

To identify the potential biomarker for NOA patients, we selected eight tsRNAs which showed significant differences in expression when compared with OA patients (difference of more than three-fold change; $\mathrm{P}$ $<0.05$, examined by both negative binomial distribution and student's t-test). Moreover, these eight miRNAs were further validated individually in subsequent samples (20 NOA samples, 15 OA samples) by RT-qPCR, although only four of them (tRF-Pro-AGG-005, tRF-Val-AAC-010, tRF-Pro-AGG-003 and tRF-GlyCCC-002) significantly differentially expressed between NOA patients and OA patients (Fig 3A), the expression values of these four tsRNAs resulted in good predictive accuracy (tRF-Pro-AGG-005 (AUC 0.98 ,sensitivity $=95 \%$, specificity $=80 \%, P<0.0001$ ); tRF-Val-AAC-010 (AUC: 0.96 , sensitivity $=95 \%$, specificity $=80 \%, P<0.0001$ ); tRF-Pro-AGG-003 (AUC: 0.96, sensitivity $=95 \%$, specificity $=87 \% P<0.0001$ ); tRF-Gly-CCC-002 (AUC: 0.73 , sensitivity $=85 \%$, specificity $=60 \%, P=0.02)$ ) (Fig. 2B). As a comparison, the ROC curve analysis of testicular volume and blood FSH levels which was the classic predictor for the NOA patients was also determined, it was found that tRF-Pro-AGG-005, tRF-Val-AAC-010, tRF-Pro-AGG-003 as NOA predictors had better predictive accuracy than the testicular volume and blood FSH levels (Fig. 2B). In this study, eight miRNAs were also validated in 10 healthy samples and $5 \mathrm{IO}$ samples, we found that tRF-Val-AAC-010 and tRF-Pro-AGG-003 expression levels were significantly increased when compared 
with that from OA samples and healthy samples (Fig 3C) and tRF-Val-AAC-010 and tRF-Pro-AGG-003 expression levels in idiopathic oligospermia (IO) samples were in the middle of NOA and OA samples. These results indicate that tRF-Val-AAC-010 and tRF-Pro-AGG-003 are associated with spermatogenesis status.

We further explored whether the target genes of these tsRNAs are associated with spermatogenesis. We found 24 target genes of tRF-Val-AAC-010 and tRF-Pro-AGG-003 enriched at least four-fold higher than mRNA level in the testis compared to any other tissue (Supplementary Table 1). The 24 genes were employed for DAVID gene ontology (GO) and GAD disease enrichment analysis. GO term biological process categories showed that the target genes are involved in spermatogenesis, GAD_DISEASE analysis indicates the target gene-related diseases were azoospermia, oligospermia, and male infertility (Fig. 4A). These results further confirm that tRF-Val-AAC-010 and tRF-Pro-AGG-003 are involved in the regulation of spermatogenesis and can be used as markers to predict NOA patients. To better understand the functions and the corresponding genetic regulatory networks of tRF-Val-AAC-010 and tRF-Pro-AGG003, the gene regulatory network was visualized by the software CytoScape[26] (Fig 4B). Moreover, consistent with the above results from seminal plasma, tsRNAs examined in the testis from NOA patients and OA patients showed that the tRF-Val-AAC-010 and tRF-Pro-AGG-003 levels in NOA patients were also higher than those in OA patients, indicating that the tRF-Val-AAC-010 and tRF-Pro-AGG-003 expression levels can reflect the individual testicular tissue pathological status and therefore could serve as a novel biomarker for NOA diagnosis.

\section{Multifactor models for diagnosis of non-obstructive azoospermia}

Although tRF-Val-AAC-010 and tRF-Pro-AGG-003 have a better predictive accuracy than the classic predictor of blood FSH level and testicular volume, none of them have a $100 \%$ sensitivity and $100 \%$ specificity (Fig 5A). We found that the combination of tRF-Val-AAC-010 and tRF-Pro-AGG-003 and the combination of tRF-Val-AAC-010, tRF-Pro-AGG-003 and blood FSH level can both completely discriminate NOA patients from OA patients with $100 \%$ sensitivity and $100 \%$ specificity (Fig $5 B-C$ ). As a comparison, the discriminability of the combination of testicular volume and blood FSH levels which was the classic predictor for NOA patients was also determined and it was found that the combination of testicular volume and blood FSH levels can not completely discriminate NOA patients from OA patients and healthy people (Fig 5D). A binomial regression equation obtained from the generalized linear model (GLM) was then presented by using the tRF-Val-AAC-010 and tRF-Pro-AGG-003 expression levels to calculate multiple-factor risk score for predicting NOA patients. The multiple-factor risk score was equal to $\exp (321.9$ - 166.1 $\times$ tRF-Pro-AGG-003-234.5 × tRF-Val-AAC-010) / (1 + exp(321.9 - $166.1 \times$ tRF-Pro-AGG003-234.5 $\times$ tRF-Val-AAC-010)). If the multiple-factor risk score is less than 0.5 , the patient can be diagnosed as non-obstructive azoospermia; if the result is greater than 0.5 , the patient can be diagnosed as obstructive azoospermia.

\section{Discussion}


For NOA and OA patients, testicular sperm extraction (TESE) is an important means to obtain sperm, however, routine indices such as testicular volume and blood FSH level have limited clinical application for predicting the outcome of TESE for azoospermia[4]. Recent studies have shown that seminal plasma contains high concentrations of exosomes, which come from different tissue cells in the male reproductive tract[3]. So the exosomes in seminal plasma can be used as a means of selective transport and delivery of various regulatory molecules to the seminal plasma then can reflect the pathophysiological status of the testis. Several studies also found that tsRNA is enriched in seminal plasma and closely related to spermatogenesis[14, 27], and tsRNAs are more stable than linear RNA because they are not easily degraded, so they can be used as non-invasive diagnostic biomarkers for NOA.

Seminal plasma exosome tsRNAs as NOA biomarkers have not been reported yet. In this study, we first report the characteristics of tsRNA detected in seminal plasma exosomes from NOA patients, OA patients, and healthy people by deep sequencing. 500 known tsRNAs and 300 novel tsRNAs were detected in this study, and we aimed to explore the potential value of exosomal tsRNAs for NOA diagnosis by using these tsRNA profiles. Since both OA patients and healthy people have normal spermatogenesis, we believe that if tsRNAs are used as markers for diagnosis of NOA, they should have a significant differential expression between NOA patients and OA patients, and they should also show a significant difference between NOA patients and healthy people but not OA patients and healthy people. We found that tRF-ValAAC-010 and tRF-Pro-AGG-003 meet this condition. At the same time, these two tsRNAs were also differentially expressed in the testis of NOA patients and OA patients, which further indicated that tRF-ValAAC-010 and tRF-Pro-AGG-003 in seminal exosomes could be important markers in the diagnosis of NOA.

Another condition for tsRNAs to be good clinical biomarkers for NOA is that they should be involved in the development of NOA. Since tsRNAs play a role in the biological process mainly through target genes, therefore, we then predicted the target genes of tRF-Val-AAC-010 and tRF-Pro-AGG-003 and found that 24 targets genes were significantly enriched in the testicles (At least four-fold higher mRNA level in the testis compared to any other tissue). Further analysis of the GO terms for the 24 target genes showed that its biological process is significantly enriched in spermatogenesis, and the use of GAD_DISEASE function analysis showed that tRF-Val-AAC-010 and tRF-Pro-AGG-003 were also enriched in sperm in the absence of disease. The observations in our study suggest that tRF-Val-AAC-010 and tRF-Pro-AGG-003 might be involved in the biological process of spermatogenesis. Taken together, the first comprehensive assessment of the exosome tsRNAs expression in diagnosis of NOA patients and OA patients and subsequent functional prediction, provides strong evidence for further etiology study of NOA.

Previous work showed that multiple variations which single has small effect sizes in diseases can improve risk prediction for many diseases $[17,18]$. This study identified tRF-Val-AAC-010 and tRF-ProAGG-003 combinations, which showed a good prediction of NOA with $100 \%$ sensitivity and $100 \%$ specificity. General linear model (GLM) that is conventionally used to comprehend genetic epistasis was first used to identify biomarker relationships. Since the expressions of tsRNAs are active, it is difficult to define the cut off value in the case of multiple biomarkers. Therefore, the binomial regression equation 
which can be used to calculate the multiple-factor risk score fitted from GLM was valuable for clinical utility.

\section{Conclusions}

This study first evaluated the potential of tsRNAs as promising biomarkers for NOA diagnosis and identified exosomal tRF-Val-AAC-010 and tRF-pro- AGG-003 in seminal plasma which are valuable biomarkers for NOA diagnosis. We also found that the combinations of tRF-Val-AAC-010 and tRF-pro AGG-003 have a better discriminating ability between NOA patients and OA patients than a single biomarker. We further predicted the target genes of the tRF-Val-AAC-010 and tRF-pro -AGG-003 and conducted functional and gene regulatory network analysis, providing strong evidence for further etiological studies.

Although our study suggests that tRF-Val-AAC-010 and tRF-pro-AGG-003 can be used as a biomarker for predicting the presence of sperm in testicular tissue, they should be validated in large samples from different countries or regions before clinical application.

\section{Abbreviations}

tsRNA: tRNA-derived small RNA

NOA: non-obstructive azoospermia

OA: obstructive azoospermia

IO: idiopathic oligoasthenospermia

TESE: testicular sperm extractions

tRF: tRNA related fragments

AUC: Area Under ROC Curve

GLM: Generalized linear model

\section{Declarations}

\section{Ethics approval and consent to participate}

This study was approved by the Research Ethics Commission of Xuzhou Central Hospital (XZXY-LJ20200721-028), and written informed consent was obtained from all participants.

\section{Consent for publication}


All the authors and participants have approved the manuscript for publication.

\section{Availability of data and materials}

The datasets used and/or analyzed during the current study are available from the corresponding author on reasonable request.

\section{Competing interests}

All other authors declare that they have no competing interests.

\section{Funding}

This work was supported by the National Natural Science Foundation of China $(81701390,81902040)$, Natural Science Foundation of Jiangsu Province (BK20170250), Xuzhou science and technology innovation project (KC19057) and Xuzhou Health Innovation Team (XWCX201603).

\section{Authors' contributions}

$\mathrm{ZZ}$ and $\mathrm{CH}$ designed the experiments; $\mathrm{XH}, \mathrm{YL}, \mathrm{YZ}, \mathrm{QZ}, \mathrm{ZL}$ and $\mathrm{HC}$ performed the experiments; $\mathrm{ZZ}, \mathrm{XH}, \mathrm{YL}$ and $\mathrm{LW}$ performed statistical analysis; $\mathrm{HC}, \mathrm{LH}, \mathrm{FH}$ and $\mathrm{YC}$ collected clinical samples and analyzed clinical data; $\mathrm{ZZ}$ and $\mathrm{CH}$ wrote the manuscript.

\section{Acknowledgements}

The experiments in this article were completed in Public Experimental Research Center of Xuzhou Medical University and we thanks the teachers for their support and help during the experiments.

\section{References}

1. Philippov OS, Radionchenko AA, Bolotova VP, Voronovskaya NI, Potemkina TV. Estimation of the prevalence and causes of infertility in western Siberia. Bull World Health Organ. 1998;76:183-7.

2. Olesen IA, Joensen UN, Petersen JH, Almstrup K, Rajpert-De Meyts E, Carlsen E, et al. Decrease in semen quality and Leydig cell function in infertile men: a longitudinal study. Hum Reprod. 2018;33:1963-74.

3. Barcelo M, Mata A, Bassas L, Larriba S. Exosomal microRNAs in seminal plasma are markers of the origin of azoospermia and can predict the presence of sperm in testicular tissue. Hum Reprod. 2018;33:1087-98.

4. McLachlan RI, Rajpert-De Meyts E, Hoei-Hansen CE, de Kretser DM, Skakkebaek NE. Histological evaluation of the human testis-approaches to optimizing the clinical value of the assessment: mini review. Hum Reprod. 2007;22:2-16.

5. Ramasamy R, Yagan N, Schlegel PN. Structural and functional changes to the testis after conventional versus microdissection testicular sperm extraction. Urology. 2005;65:1190-4. 
6. Godoy PM, Bhakta NR, Barczak AJ, Cakmak H, Fisher S, MacKenzie TC, et al. Large Differences in Small RNA Composition Between Human Biofluids. Cell Rep. 2018;25:1346-58.

7. Zheng LL, Xu WL, Liu S, Sun WJ, Li JH, Wu J, et al. tRF2Cancer: A web server to detect tRNA-derived small RNA fragments (tRFs) and their expression in multiple cancers. Nucleic Acids Res. 2016;44:W185-93.

8. Kumar P, Kuscu C, Dutta A. Biogenesis and Function of Transfer RNA-Related Fragments (tRFs). Trends Biochem Sci. 2016;41:679-89.

9. Li S, Xu Z, Sheng J. tRNA-Derived Small RNA: A Novel Regulatory Small Non-Coding RNA. Genes (Basel). 2018;9.

10. Geslain R, Eriani G. Regulation of translation dynamic and neoplastic conversion by tRNA and their pieces. Translation (Austin). 2014;2:e28586.

11. Zhu L, Li J, Gong Y, Wu Q, Tan S, Sun D, et al. Exosomal tRNA-derived small RNA as a promising biomarker for cancer diagnosis. Mol Cancer. 2019;18:74.

12. Quek C, Bellingham SA, Jung CH, Scicluna BJ, Shambrook MC, Sharples RA, et al. Defining the purity of exosomes required for diagnostic profiling of small RNA suitable for biomarker discovery. RNA Biol. 2017; 14:245-58.

13. Huttner E, Mergner U, Braun R, Schoneich J. Increased frequency of 6-thioguanine-resistant lymphocytes in peripheral blood of workers employed in cyclophosphamide production. Mutat Res. 1990;243:101-7.

14. Sharma U, Conine CC, Shea JM, Boskovic A, Derr AG, Bing XY, et al. Biogenesis and function of tRNA fragments during sperm maturation and fertilization in mammals. Science. 2016;351:391-6.

15. Couvillion MT, Sachidanandam R, Collins K. A growth-essential Tetrahymena Piwi protein carries tRNA fragment cargo. Genes Dev. 2010;24:2742-7.

16. Kowalczykiewicz D, Swiercz A, Handschuh L, Lesniak K, Figlerowicz M, Wrzesinski J. Characterization of Sus scrofa small non-coding RNAs present in both female and male gonads. PLoS One. 2014;9:e113249.

17. Zhu Z, Yuan D, Luo D, Lu X, Huang S. Enrichment of Minor Alleles of Common SNPs and Improved Risk Prediction for Parkinson's Disease. PLoS One. 2015;10:e0133421.

18. Curtis D. Clinical relevance of genome-wide polygenic score may be less than claimed. Ann Hum Genet. 2019;83:274-7.

19. Song L, Langfelder P, Horvath S. Random generalized linear model: a highly accurate and interpretable ensemble predictor. BMC Bioinformatics. 2013;14:5.

20. Zhang S, Li H, Zheng L, Li H, Feng C, Zhang W. Identification of functional tRNA-derived fragments in senescence-accelerated mouse prone 8 brain. Aging (Albany NY). 2019;11:10485-98.

21. Kechin A, Boyarskikh U, Kel A, Filipenko M. cutPrimers: A New Tool for Accurate Cutting of Primers from Reads of Targeted Next Generation Sequencing. J Comput Biol. 2017;24:1138-43. 
22. Langmead B, Trapnell C, Pop M, Salzberg SL. Ultrafast and memory-efficient alignment of short DNA sequences to the human genome. Genome Biol. 2009;10:R25.

23. Friedlander MR, Mackowiak SD, Li N, Chen W, Rajewsky N. miRDeep2 accurately identifies known and hundreds of novel microRNA genes in seven animal clades. Nucleic Acids Res. 2012;40:37-52.

24. Robinson MD, McCarthy DJ, Smyth GK. edgeR: a Bioconductor package for differential expression analysis of digital gene expression data. Bioinformatics. 2010;26:139-40.

25. Durdevic Z, Schaefer M. tRNA modifications: necessary for correct tRNA-derived fragments during the recovery from stress? Bioessays. 2013;35:323-7.

26. Shannon P, Markiel A, Ozier O, Baliga NS, Wang JT, Ramage D, et al. Cytoscape: a software environment for integrated models of biomolecular interaction networks. Genome Res. 2003;13:2498-504.

27. Peng H, Shi J, Zhang Y, Zhang H, Liao S, Li W, et al. A novel class of tRNA-derived small RNAs extremely enriched in mature mouse sperm. Cell Res. 2012;22:1609-12.

\section{Figures}

A

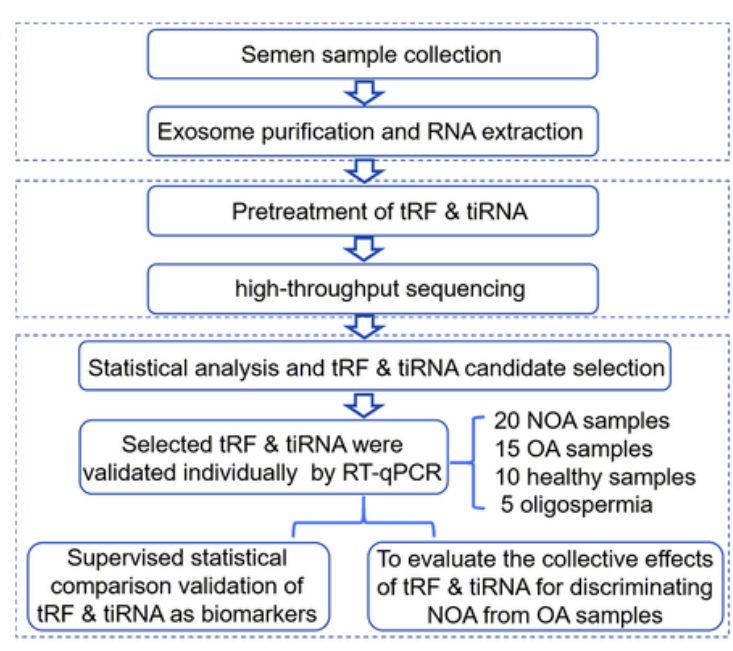

$\mathrm{F}$

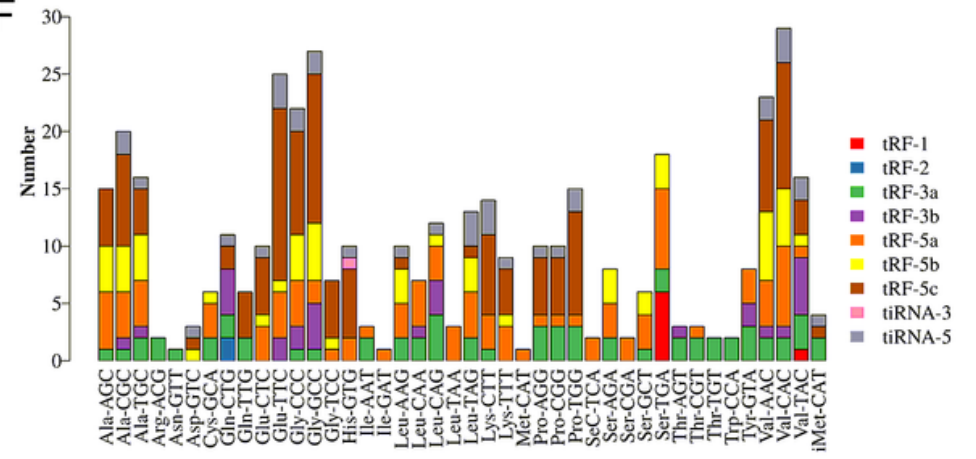

B

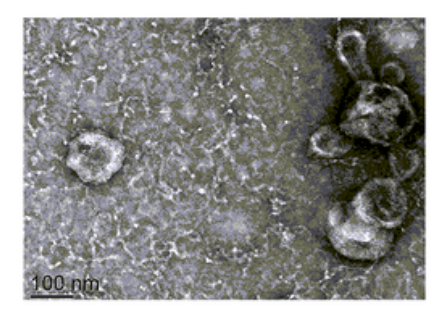

C

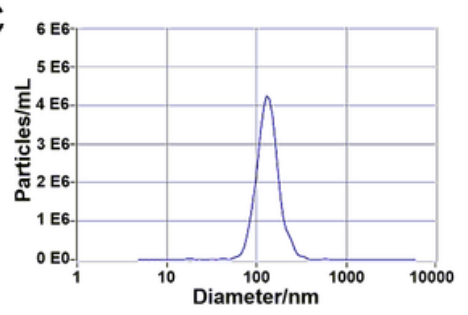

D

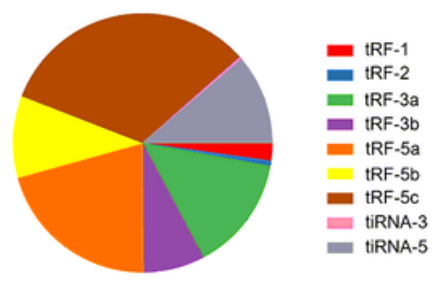

E

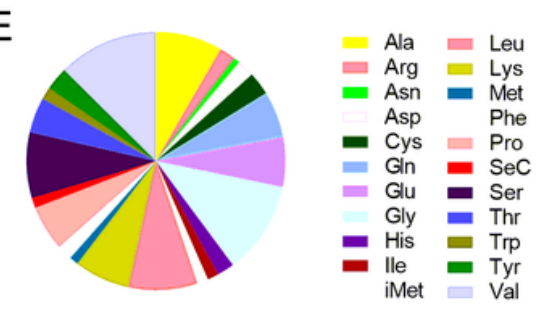

Figure 1

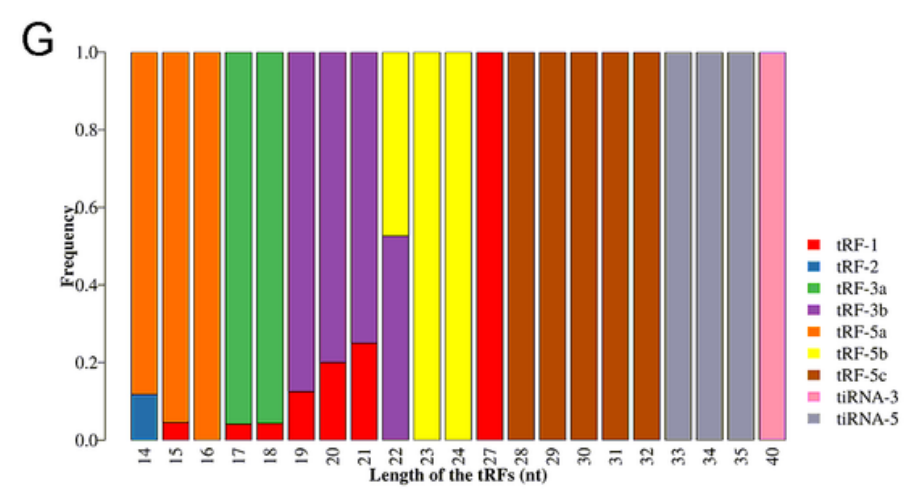


Profiling of exosomal tsRNAs in seminal plasma. (A) Flow chart of the two stages of tsRNA profiling and validation performed in the study. (B) Transmission electron micrograph (TEM) of exosomes isolated from seminal plasma (The scale bar is $100 \mathrm{~nm}$ ). (C) nanoparticle tracking analysis of exosomes isolated from seminal plasma. (D) Pie char of the distribution of subtype tsRNAs. The values in bracket are represented the number of subtype tsRNAs. The color represents the subtype tsRNAs. (E) Pie char of the distribution of the origin for tsRNAs in seminal plasma. (F) The number of subtype tsRNAs against tRNA isodecoders. The $X$ axes represents tRNA isodecoders and the $Y$ axes show the number of all subtype tsRNAs against tRNA isodecoders. The color represents the subtype tsRNAs. (G) The Frequency of Subtype against Length of the tRF \& tiRNA. The $X$ axes represents length of tRF \& tiRNA and the $Y$ axes show the frequency of the subtype against length of tsRNAs. The color represents the subtype tsRNAs.
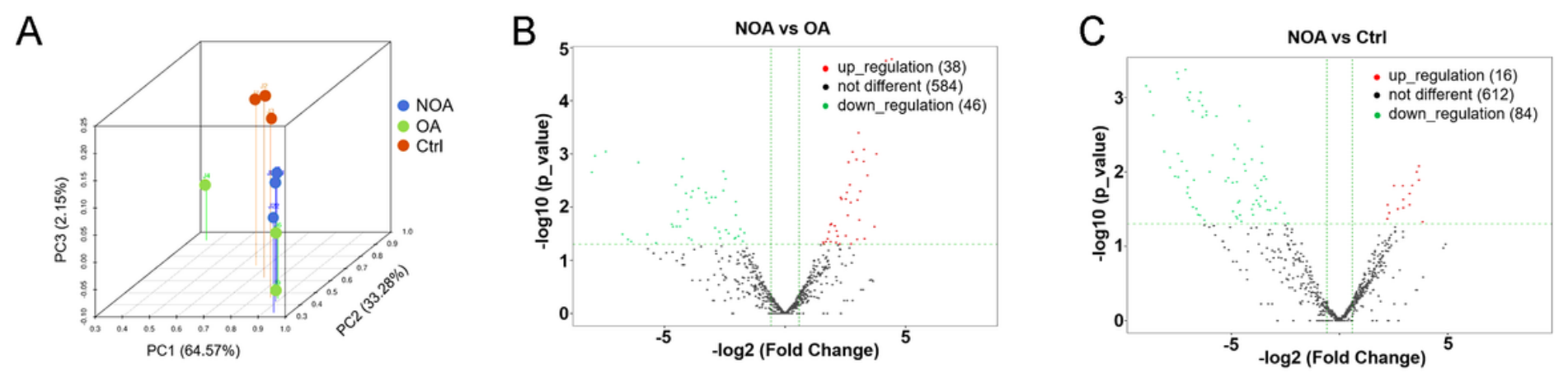

\section{Figure 2}

The analysis of expression level in NOA, OA and healthy samples. (A) Primary component analyze. the X, $Y$ and $Z$ axis represents the three main factors which affected the expression level of the sample. The colored point represents the corresponding sample, and the location of it shows the main character of the sample. Space distance represents the similarity of data size. The volcano plot of differentially expressed tsRNAs between NOA and OA patients (B) and between NOA patients and healthy people (C). The values of $X$ and $Y$ axes in the volcano plot are log 2 transformed fold change and -log 10 transformed $p$-values between the two groups, respectively. Red/Green circles indicate statistically significant differentially expressed tsRNAs with fold change no less than 1.5 and p-value $\leqslant 0.05$ (Red: up-regulated; Green: downregulated). Gray circles indicate non-differentially expressed tsRNAs, with FC and/or q-value are not meeting the cutoff thresholds. 

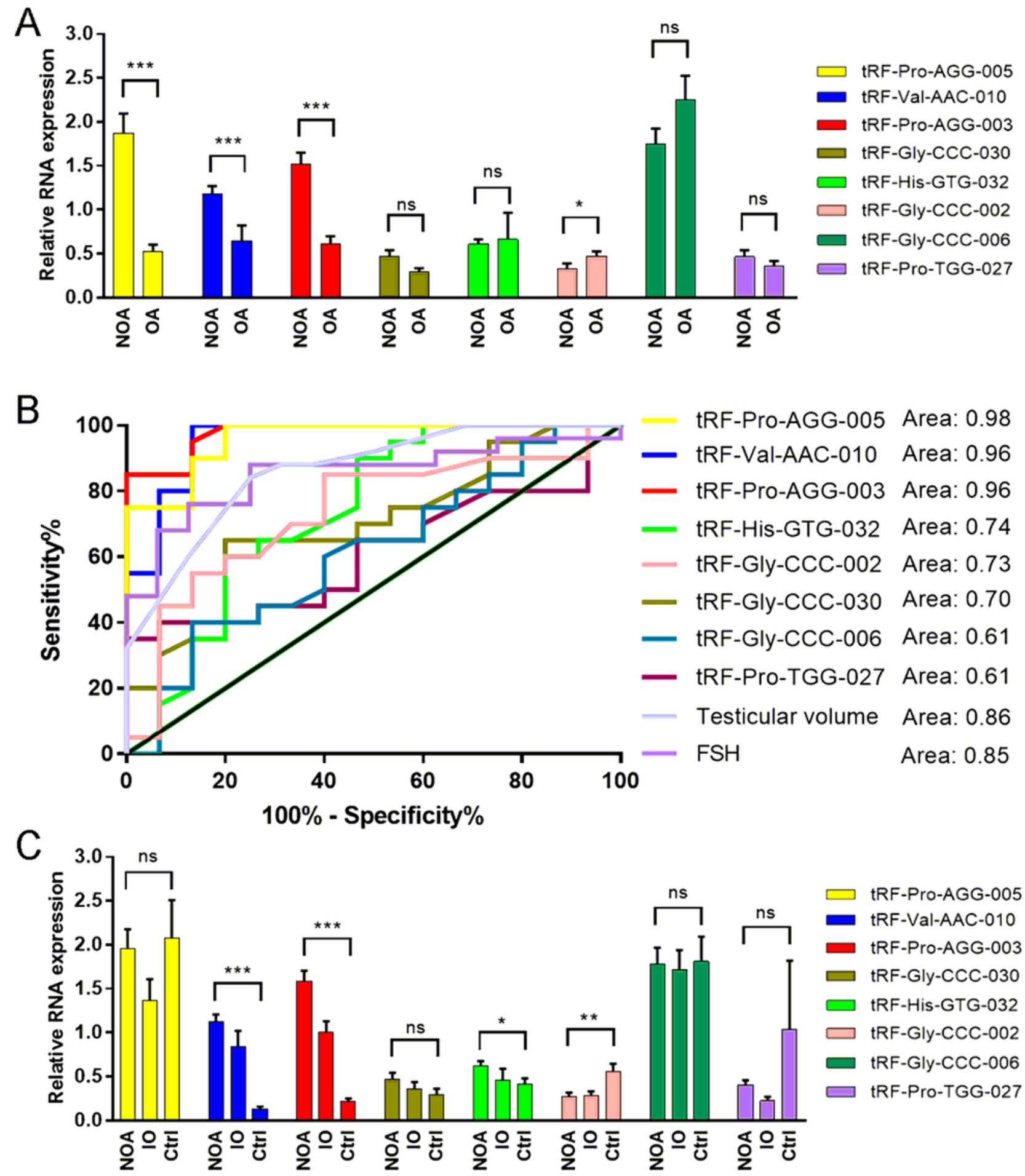

Figure 3

Validation of the eight selected tsRNAs and to assess their predictive efficiency for distinguishing NOA patients with spermatogenic failure from OA patients. (A) Validation of the eight selected tsRNAs by qRTPCR from NOA and OA. (B) ROC curve of the eight selected tsRNAs for predictive classification of azoospermic patients into NOA and OA subtypes. (C) Validation of the eight selected tsRNAs by qRT-PCR from NOA, IO and healthy samples. Normalized expression levels relative to the U6 gene. Data shown as 
the means \pm SEM. Statistical analysis was performed by using two-tailed unequal variant Student's t-test (*P-value $<0.05 ; * \star$ P-value $<0.01 ; * \star \star P-v a l u e<0.001$ ).

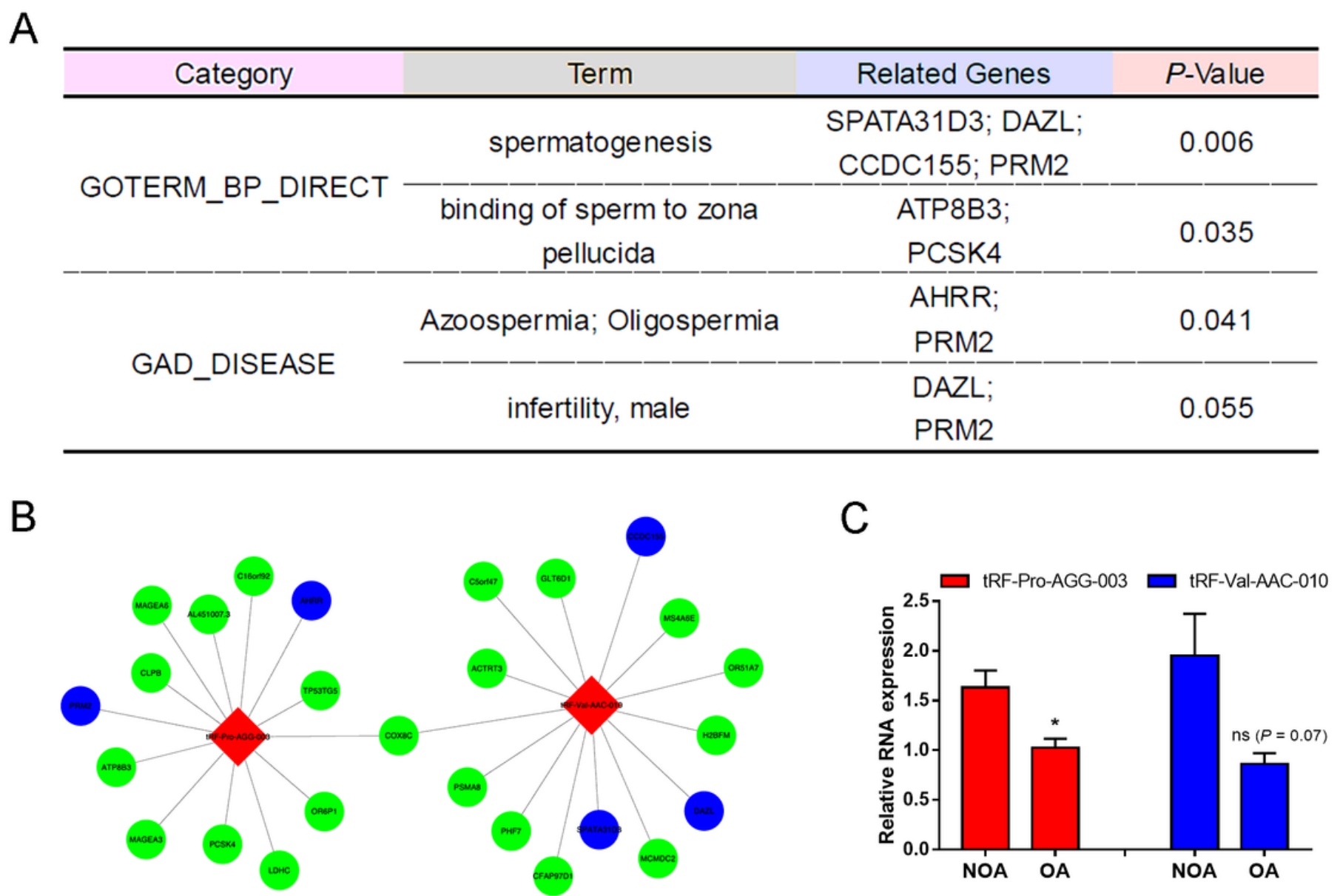

\section{Figure 4}

tRF-Val-AAC-010 and tRF-Pro-AGG-003 are the biomarkers of NOA and involved in spermatogenesis. (A) The most significant biological process $G 0$ terms and GAD diseases for the 24 target genes of tRF-ValAAC-010 and tRF-Pro-AGG-003. (B) The tsRNA/mRNA network analysis. The network included the two candidate tsRNAs and their predicted target mRNAs (Nodes in red diamond are tsRNAs; nodes in circle color are predicted target mRNAs fot the two tsRNA; nodes of the blue circles are genes that have been reported to be involved in spermatogenesis). (D) Relative expression of tRF-Val-AAC-010 and tRF-ProAGG-003 in exosomes from azoospermia testis. Data shown as the means \pm SEM. Statistical analysis was performed by using two-tailed unequal variant Student's t-test ( ${ }^{*}$-value $<0.05$ ). 

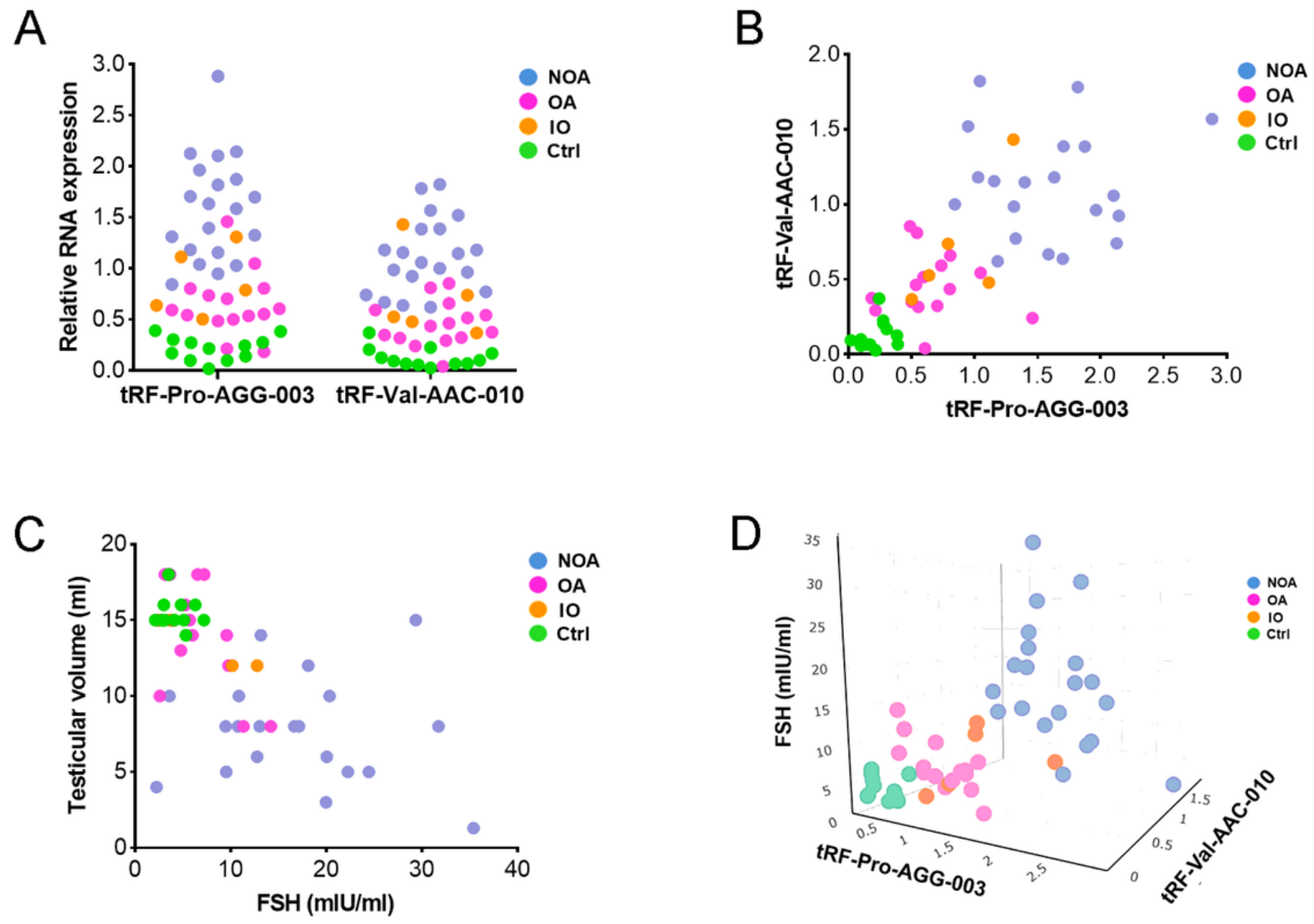

Figure 5

The predictive efficiency of single factor and multifactor models for distinguishing NOA patients with spermatogenic failure from $\mathrm{OA}, \mathrm{IO}$ and healthy samples. The predictive efficiency of tRF-Val-AAC-010 and tRF-Pro-AGG-003 (A); the combination of tRF-Val-AAC-010 and tRF-Pro-AGG-003; the combination of testicular volume and blood FSH level (C); and the combination of blood FSH level, tRF-Val-AAC-010 and tRF-Pro-AGG-003 (D); for distinguishing NOA patients with spermatogenic failure from OA, IO and healthy samples.

\section{Supplementary Files}

This is a list of supplementary files associated with this preprint. Click to download.

- SupplementaryTable2.xlsx

- SupplementaryTable1.xlsx 